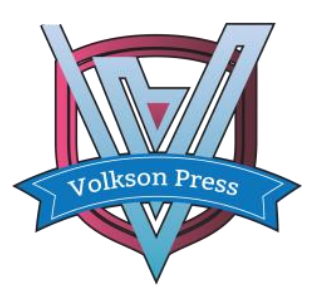

Contents List available at VOLKSON PRESS

Economics \& Management Innovations(EMI)

DOI : http://doi.org/10.26480/icemi.01.2017.171.173

\title{
An Information System for Supporting the Library Center of Academic Resources, the Judiciary Office
}

\author{
Anchaya Tuntiwechanon ${ }^{1}$, Waraporn Jirapanthong ${ }^{2, *}$ \\ ${ }^{1}$ College of Innovative Technology and Engineering, Dhurakij Pundit University, Thailand \\ ${ }^{2}$ College of Creative Design and Entertainment Technology, Dhurakij Pundit University, Thailand \\ *waraporn.jir@dpu.ac.th \\ This is an open access article distributed under the Creative Commons Attribution License, which permits unrestricted use, distribution, and reproduction in any \\ medium, provided the original work is properly cited.
}

\section{ARTICLE DETAILS}

\section{Article History:}

Received 02 october 2017 Accepted 06 october 2017 Available online 11 october 2017

\section{Keywords:}

Information Management System, Digital System, Library Center..

\section{ABSTRACT}

The paper presents the development of the information system for supporting the justice court libraries. The objectives focus on the improvement of the existing system in term of efficiency and usability. The new system provides main functions supporting intelligent search and also facilitates the use of information resources in the organization. The new system is aimed to be used straightforwardly and support the decision making. The system architecture is based on web-based application which allows users to access though the Internet. The architecture is designed and implemented based on Object-Oriented Programming (OOP) and MySQL Workbench. Also the tool, NetBeans and Jasper are applied. We have simulated a set of testing data to evaluate the system performance and competency, particularly in term of accuracy, efficiency, and recall.

\section{Introduction}

The center for academic resources, under the office of Judiciary, is responsible for supporting research, data analysis about governmental policies, legal, including information services. They provide the standards, rules, procedures, and guidelines of information services for the Judiciary office. They also conduct the provision of books, periodicals, publications, documents, audio-visual materials that collect the academic information modern law and information resources. However, the main problem of the existing system is that it can allow users to manage and access the information resources at site only. Also, the system supports the services with small data storage and low efficiency. The main objectives of this work are to: i) to analyse and design an information system to support the knowledge integration between justice libraries in Thailand, ii) to improve theefficiency and competency of the usage of existing database, iii) to develop the innovation of library services, and iv) to support decision making for justice executives from different regions in Thailand. The work is expected to increase the efficiency and effectiveness of information retrieval for the justice courts in Thailand, also to reduce some operation and maintenance cost.

\section{Background and Related Work}

\subsection{Object-Oriented System Analysis and Design}

Object Oriented Methodology is a new system development approach, encouraging and facilitating re-use of software components. It employs the international standard, Unified Modeling Language (UML) from the Object Management Group (OMG). Using this methodology, a system can be developed on a component basis, which enables the effective re-use of existing components. It facilitates the sharing of its other system components. Object Oriented Methodology asks the analyst to determine what the objects of the system are, what responsibilities and relationships an object has to do with the other objects, and how they behave over time. There are three types of Object Oriented Methodologies: i) Object Modeling Techniques (OMT), ii) Object Process Methodology (OPM), and

iii) Rational Unified Process (RUP).

Firstly, Object Modeling Techniques (OMT) was one of the first object oriented methodologies and was introduced by Rumbaugh in 1991[5].
OMT uses three different models that are combined in a way that is analogous to the older structured methodologies. With OMT, the main goal of the analysis is to build models of the world. The requirements of the users, developers and managers provide the information needed to develop the initial problem statement. OMT Models appear in one of those three models which are: a) Object Model that depicts the object classes and their relationships as a class diagram, which represents the static structure of the system and observes all the objects as static and does not pay any attention to their dynamic nature, b) Dynamic Model that captures the behavior of the system over time and the flow control and events in the EventTrace Diagrams and State Transition Diagrams, which portrays the changes occurring in the states of various objects with the events that might occur in the system, and c) Functional Model that describes the data transformations of the system, which describes the flow of data and the changes that occur to the data throughout the system. Afterwards, the design is performed and it specifies all of the details needed to describe how the system will be implemented. The details of the system analysis and system design are implemented. The objects identified in the system design phase are designed.

Secondly, Object Process Methodology (OPM), called as second generation methodology, was first introduced in 1995 [6]. It has only one diagram that is the Object Process Diagram (OPD) which is used for modeling the structure, function and behavior of the system. It has a strong emphasis on modeling but has a weaker emphasis on process. It consists of three main processes: a) Initiating that determines high level requirements, the scope of the system and the resources that will be required, b) Developing that involves the detailed analysis, design and implementation of the system, and c) Deploying, that introduces the system to the user and subsequent maintenance of the system.

Thirdly, Rational Unified Process (RUP) was developed in Rational Corporation in 1998 [7]. It consists of four phases which can be broken down into iterations: a) Inception, b) Elaboration, c) Construction, and d) Transition. Each iteration consists of nine work areas called disciplines. For each discipline, RUP defines a set of artefacts (work products), activities (work undertaken on the artefacts) and roles (the responsibilities of the members of the development team). 


\subsection{Unified Modeling Language (UML)}

System Analysis and Design with OOP can be expressed by UML, which is schematically defined by the standard. UML 2.0 [8] defines thirteen types of diagrams, divided into three categories: Six diagram types represent static application structure; three represent general types of behavior; and four represent different aspects of interactions: a) Structure Diagrams include the Class Diagram, Object Diagram, Component Diagram, Composite Structure Diagram, Package Diagram, and Deployment Diagram, b) Behavior Diagrams include the Use Case Diagram (used by some methodologies during requirements gathering); Activity Diagram, and State Machine Diagram, and c) Interaction Diagrams, all derived from the more general Behavior Diagram, include the Sequence Diagram, Communication Diagram, Timing Diagram, and Interaction Overview Diagram.

In particular, Use Case Diagram is a diagram that shows the behavior of the user and system's functions. It also includes a set of actors who interact with each use case. Class Diagram is a diagram that shows the class and relationships between the classes.

\subsection{Java Technologies for Web Application}

Web applications are by nature distributed applications, meaning that they are programs that run on more than one computer and communicate through a network or server. Specifically, web applications are accessed with a web browser and are popular because of the ease of using the browser as a user client. One web application can be accessed and used by millions of people. Web applications frequently require an additional markup or scripting language, such as HTML, CSS, or JavaScript programming language. Also, many applications use only the Java programming language, which is ideal because of its versatility. The Java technologies are applied to create web applications are a part of the Java EE platform, in addition to many of the Java Platform, Standard Edition (Java SE) classes and packages.

The Java Servlet API is used to define HTTP-specific classes. A servlet class extends the capabilities of servers that host applications that are accessed by way of a request-response programming model. Although servlets can respond to any type of request, they are commonly used to extend the applications hosted by web servers. Moreover, Java Server Pages (JSP) technology [9] provides a simplified, fast way to create dynamic web content. JSP technology enables rapid development of web-based applications that are server- and platform-independent. Java API for XML Processing (JAXP), part of the Java SE platform, supports the processing of XML documents using the Document Object Model (DOM), the Simple API for XML (SAX), and Extensible Stylesheet Language Transformations (XSLT). JAXP enables applications to parse and transform XML documents independent of a particular XMLprocessing implementation. It is applied with JAXP that provides namespace support. Some popular tools are supporting Java technologies such as NetBeans IDE, Sun Java Studio Creator IDE, and Sun Java Studio Enterprise IDE.

\section{Information System for the Library Center of Academic} Resources, the Judiciary Office

\subsection{Analysis and Design}

Regarding the analysis and design, the system consists of main functions: i) the system must provide the information resource management system which includes books, media, audiovisual and journals,

ii) the system must simultaneously and consistently operate the maintenance of the information resources, iii) the system must provide the information retrieval system via resource's title, author, publisher, year of publication, iv) the system must be available for all courts of Justice, v) the system must provide the services for only authenticated users, and vi) the system must collect the reports of information resource retrieval.

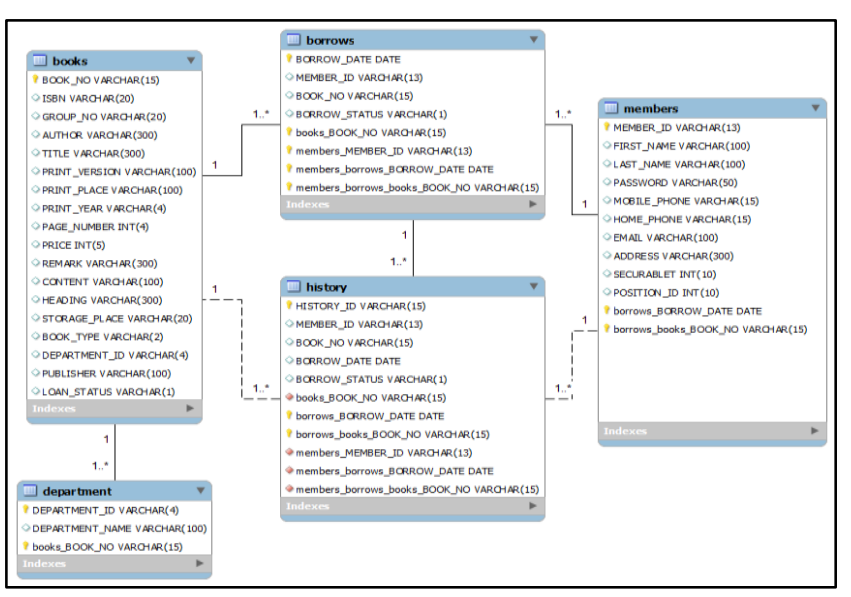

Fig. 1 Entity Relationship Diagram

We performed a focus group and elicited the system and user requirements. According to the requirement specification, the database is designed. The system architecture is also designed to support the database repository including library materials e.g. books, articles, magazines, journals, library member profiles, borrowing records as shown in Figure 1. The data repository aims to serve all justice courts.

Moreover, as shown in Figure 2, the systems provide main functions: 1) user registration, which allows only justice officers to be the system members, 2) authentication, which identifies a user and his right to use the system, 3) searching, which allows a user to search and retrieve an inquiry, 4) borrowing and returning, which allows a user to borrow and return library items, and 5) report generating, which allows a user to generate an ad-hoc reports. The users are typed as: i) members who registered and are authenticated to use the system, ii) librarians who are in charge of librarying processes, iii) guests who refer unregistered users, and iv) administrators who maintain the system.

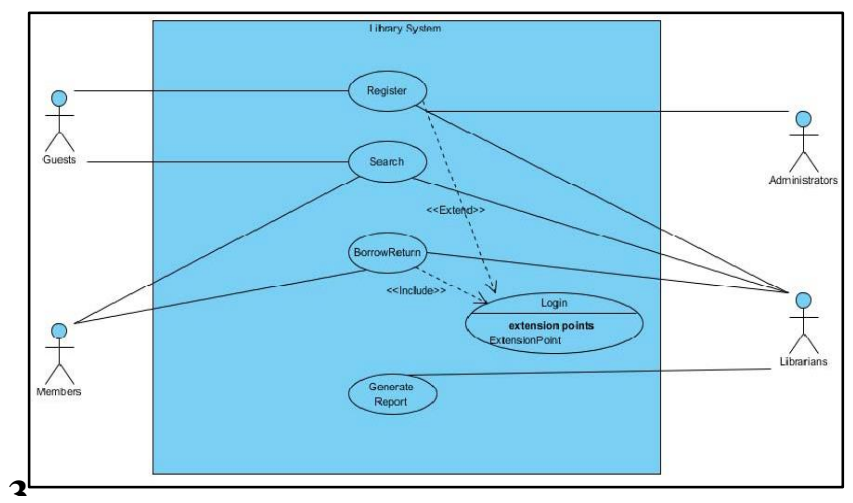

Fig. 2 Use Case Diagram

We have developed a prototype tool to facilitate the demonstration and evaluation of the approach. The main functionalities of the tool are implemented according to the design. The examples of new system are shown in Figures 3 and 4. Different cases are created to demonstrate different situations of the use of the system, involving (a) inquiring different types of library items; and (b) different sites of use.

As shown in Figure 3, the system provides a functional menu of item searching. It allows a user to search items by item code, item title, author, category, ISBN, and related keyword. The result of searching returns as a list of items that show each item's code, ISBN, court site, and status. Moreover, as shown in Figure 4, the system provides a functional menu of library item loan. It allows a user to make a loan of library items. The user must get authenticated and make a loan under defined constraints. 


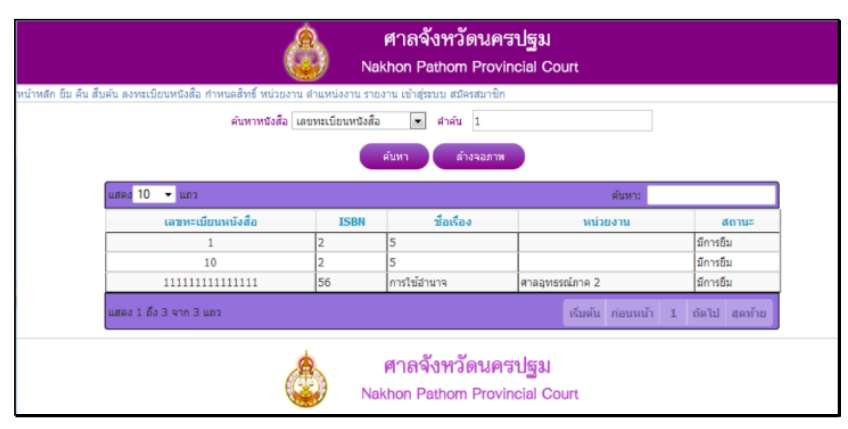

Fig. 3 A searching menu

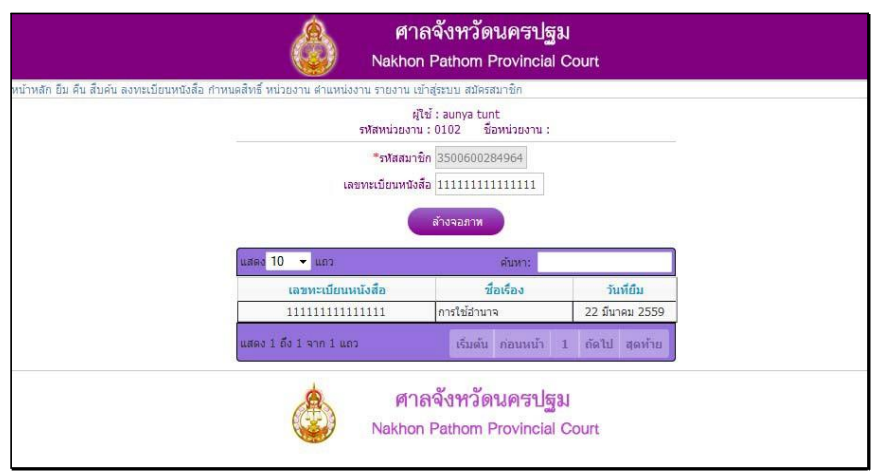

Fig. 4 An Example of New System

\section{Evaluation and Conclusions}

To evaluate the system, we created the scenarios of system use based on real situations. The participants include the Judicial officers and information technology staffs. The participants are trained regarding the usage of the new system for 2 hours. They are asked to use the system as a part of their ordinary routine work. The evaluation measurements are accuracy and efficiency of the system as shown in Table 1.

Table 1 Evaluation Result

\begin{tabular}{|l|c|}
\hline \multicolumn{1}{|c|}{ Criteria } & Result (out of 5.0) \\
\hline Ease of use & 4.0 \\
\hline Effectiveness of data management & 4.5 \\
\hline Completeness of system functions & 5.0 \\
\hline Precision of information retrival & 5.0 \\
\hline Efficiency of the system & 4.0 \\
\hline Consistency (Recall) of information retrival & 4.8 \\
\hline
\end{tabular}

Regarding the evaluation results, the use of the new system which supports the information management system for the Justice Court libraries is found easier and more straightforward. The system allows users the system use more effectively and efficiently. The experiments of information retrieval are evaluated by considering three criteria: (i) straightforward, (ii) correctness, and (iii) completeness. The results of test cases show that correctly identified is $97.93 \%$ while misidentified is $17.6 \%$. The percentage of correctly identified is high (97.93\%). It implies the new information system with high performance. The percentage of misidentifying is fairly low (17.6\%). This is due to incomplete data e.g. missing some attribute values, incorrect data. This presents the positive feedback of users and it is believed that the system would be installed and available in local justice courts in Thailand soon. In addition, some suggestions for the new system are collected for next phase of the system development, for examples, the system should provide the barcode readers in the libraries and identify the specific types of users.

A number of possible directions for further investigations have been identified. We provide in this section future work of the research, what needs to be done to improve the approach and to increase the benefits of the approach. Tools for Document Generation and Visualisation for large number of various artefacts should be implemented. It is therefore believed that the approach could benefit by providing tool fully support for the specification of documents. In addition, sophisticated techniques for visualization could support the use of documents more efficiently.

\section{References}

[1] Arendsen, A. (2548). Professional Java Development with the Spring Framework, from http://docs.spring.io/docs/Spring-MVC-step-bystep/

[2] Booch, G. (2544). The architecture of Web applications, from http://www.ibm.com/developerworks/ibm/library/it-booch web/

[3] Bannon, R., and A. Chin. (2550). MySQL Conceptual Architecture, from http://www.swen.uwaterloo.ca/ mrbannon/cs798/assignment 01/m ysql.pdf

[4] Sun Microsystems. (2550). Apache Tomcat, from http://tomcat.apache.org/ [5] Rumbaugh, J. et. al. (1990). ObjectOriented Modeling and Design. Prentice Hall. ISBN 0-13-629841-9

[6] Dov, D. (1995). Object-Process Analysis: Maintaining the Balance between System Structure and Behavior. Journal of Logic and Computation. 5 (2): 227-249. doi:10.1093/logcom/5.2.227.

[7] Kruchten, P. (1999). Rational Unified Process - An Introduction, Addison-Wesley. [8] OMG. (2005). Unified Modeling Language (UML). Available at:

http://www.omg.org/spec/UML/2.0/

[9] Oracle. (1999). JavaSever Pages. Available at: http://www.oracle.com/technetwork/java/index.html 